aim was to assess the use of Lanreotide in patients who presented with chronic gastrointestinal bleeding due to angioectasias.

Method Patients who were diagnosed to have angioectasia (gastric or small bowel) with portal hypertension at endoscopy and who received lanreotide injections were included. Aeitology of underlying portal hypertension, previous history of bleeding and endotherapy, transfusion history and any side effects of lanreotide was recorded.

Results A total of 12 patients (75\% Males, mean age 68) were identified between 2007 and 2020. Lanreotide 60mg (milligrams) given monthly, subcutaneously in all patients. Causes for portal hypertension: portal vein thrombus- 3 (25\%); alcohol related liver disease - 3 (25\%); Non-alcoholic fatty liver disease- 3 (25\%); Primary biliary cholangitis- 1(8\%), Alpha one antitrypsin deficiency - $1(8 \%)$. One patient $(8 \%)$ had both alcohol and non-alcoholic fatty liver related liver disease.

11 of the 12 patients (92\%) had oesophageal varices. All of these patients also had portal hypertensive gastropathy and at least one angioectatic lesion identified. One patient $(8 \%)$ had portal hypertensive gastropathy only. Nine patients $(75 \%)$ had prior treatment with either variceal banding, glue injection, argon plasma coagulation or interventional radiology embolisation. Two patients were also treated with a trans-jugular intrahepatic portosystemic shunt.

After treatment with lanreotide, the average total number of bleeding episodes per patient reduced from 6 to 0.8 . There was also a significant reduction in the average episodes of blood transfusions: 6 to 1 .

There were $6(50 \%)$ patients who had an episode of rebleeding (defined as drop in haemoglobin and haemodynamic instability) in the first 6 months of treatment but did not require any endoscopic therapy.

Only 1 person reported any side effects with Lanreotide (diarrhoea) and stopped treatment.

Conclusion Lanreotide is a useful adjuvant treatment to therapeutic endoscopy in patients with refractory portal hypertensive bleeding due to angioectasia. It reduces transfusion requirements, bleeding episodes and overall improves patient quality of life with a good safety profile.

\section{P058 THE IMPACT OF NATIONAL LIVER OFFERING SCHEME ON THE POST-TRANSPLANT COURSE OF PATIENTS WITH ALCOHOL RELATED LIVER DISEASE: A FRIEND OR A FOE?}

${ }^{1}$ Agimol Pradeep*, ${ }^{1}$ lan Webzell, ${ }^{1}$ Shirin Khorsandi, ${ }^{1}$ Naina Shah, ${ }^{1}$ Karen Mercer, ${ }^{1}$ Andreas Prachalias, ${ }^{1}$ Varuna Aluvihare, ${ }^{1}$ Abid Suddle, ${ }^{2}$ Katie Ramos, ${ }^{3}$ Leanne Stratton Stratton, Krishna Menon, ${ }^{1}$ Wendy Littlejohn. ${ }^{1}$ Kings College Hospital, UK; ${ }^{2}$ Derriford Hospital, UK; ${ }^{3}$ Royal Victoria Hospital, Belfast, UK

\subsection{6/gutjnl-2021-BASL.67}

Introduction The National Liver Offering Scheme (NLOS) was introduced in March 2018, this is based upon the scoring system called the Transplant benefit score (TBS) to achieve best outcomes in the adult recipients.

Aim To ascertain the impact of the TBS on the transplant waiting list and Alcohol relapse rates in patients transplanted for Alcohol related Liver disease (ArLD).

Methods This is a retrospective single centre study comparing patients in pre-TBS (March 2016-March 2018) and the post-
TBS (March 2018-March 2020) period undergoing Liver transplantation (LT) for ArLD. Data were extracted from a prospectively maintained Liver transplant database. The data were analysed using the non parametric t test.

Results In the pre-TBS period, $\mathrm{n}=76$ patients underwent LT for ArLD and in the post TBS, $\mathrm{n}=94.14 \%$ (11/76) patients in the pre-TBS and 9\% (9/94) patients in the post-TBS group relapsed to Alcohol. The median waiting time on the transplant list of the patients who relapsed was $70(29 \pm 271)$ days and in the post-TBS was $8(6 \pm 50)$ days, $p=0.02$. Time to relapse in the pre-TBS period was $425(240 \pm 810)$ days and in the post-TBS was $328(136 \pm 405)$ days, $p=n s .2 / 11$ died in the pre-TBS group due to the recurrence of ArLD and 4/9 died in the post-TBS group, two of these had recurrence of ArLD

Conclusion The introduction of TBS has certainly had a positive impact by leading to a shorter transplant wait time for recipients with ArLD. Though the time to relapse appeared shorter in the post-TBS group, it did not appear to produce any significant increase in mortality compared to the pre-TBS group. Further long-term data is required to ascertain the influence of waiting time on the risk of relapse and prognosis.

However, robust screening and timely intervention is required to reduce the risk of Alcohol relapse. The utility of digital tools in the remote monitoring of Alcohol use disorder needs to be explored for early identification of Alcohol relapse.

\section{P059 THE IMPACT OF THE COVID-19 PANDEMIC ON HEPATOCELLULAR CARCINOMA DIAGNOSIS AND STAGE AT PRESENTATION IN THE WEST OF SCOTLAND}

${ }^{1}$ Cal Molloy*, ${ }^{2}$ Rachael Swann, ${ }^{2}$ Matthew Priest. 'University of Glasgow, Glasgow, UK; ${ }^{2}$ Queen Elizabeth University Hospital, Glasgow, UK

\subsection{6/gutjnl-2021-BASL.68}

Introduction Hepatocellular carcinoma (HCC) is a disease with increasing incidence in Scotland and a high mortality rate. Prior studies have shown that early diagnosis of HCC is crucial to a successful outcome. Although the 2020 the COVID19 pandemic had a global impact on all aspects of healthcare, the effect on HCC diagnosis and management has not previously been quantified.

Methods A retrospective review of the electronic patient records of all patients discussed at the weekly West of Scotland (WOS) HCC Multidisciplinary Team (MDT) meetings, with a new confirmed diagnosis of HCC. Those discussed between the periods of March 1st and September 30th of 2019 and 2020 were included.

Electronic patient records were reviewed using NHS Greater Glasgow and Clyde's online clinical platform Portal, and database TrakCare Live. Information gathered included: gender, age, liver history, Health Board, route of diagnosis, date of referral to MDT, date of MDT discussion, treatment type, and date of first treatment. MDT reports were used to collate clinical data including: performance status, Child-Pugh class, AFP levels, tumour size, tumour number, and status of extrahepatic disease. (table 1).

Results Data was collected from 99 patients in total. The 2019 cohort included 62 patients, while the 2020 cohort included 37 patients. There was a $40 \%$ reduction in total 